\title{
Sugar content of yogurt products in the UK: A comparison between 2016 and 2019
}

\author{
Eimear Sutton, Neil Hancock and J. Bernadette Moore \\ School of Food Science and Nutrition, University of Leeds, Leeds, United Kingdom
}

\begin{abstract}
Yogurt is a staple part of children's diets and is perceived as a nutrient dense food for adults and children. However, yogurts are also a significant source of free sugars for children and are a target of the UK government's sugar reduction programme, which has recommended a $20 \%$ reduction in the sugar content of yogurt and fromage frais products by 2020 . With this in mind, in November 2016 , we undertook a comprehensive survey of the nutrient contents of yogurt products in UK supermarkets (Moore et al. 2018). The aim of this work was to reassess the UK supermarkets yogurt products in 2019 and determine whether the sugar contents of yogurt products have been reduced. Product data was collected from the UK's top five online supermarkets in January 2019 using the search terms 'yogurt' and 'yoghurt'. Products were placed systematically into the same 8 categories used in 2016: children's, drinks, dairy alternatives, organic, natural/Greek, fruit, flavoured, desserts. Products lists were collected, refined and compared to the 2016 database. All data was double-checked independently and 5\% of all entries were randomly selected and verified. GraphPad Prism V/7.0c was used for statistical analysis. After de-duplication of products found in multiple supermarkets, the 2019 database contained 893 unique products in line with the 898 surveyed in 2016. Of these, $539(60.4 \%)$ yogurts were in common (same brand and name) with the 2016 products and 354 were new, demonstrating dynamic turnover in available yogurt products during the 26 months between surveys. In comparing the total sugar contents of the 539 paired products, notably the median [range] of total sugar contents in 2019 was significantly lower than in $2016(10.8 \mathrm{~g} / 100 \mathrm{~g}$ [0.4, 29.5] versus $11.8 \mathrm{~g} / 100 \mathrm{~g}$ [0.1, 31.8]; $P<0.0001$, Wilcoxon matched-pairs signed rank test). Indeed, when all products were compared there was a significant reduction in the median total sugar in 2019 compared to 2016 (10.4g/100 g [0-32.9] versus 11.9g/100 g [0.1-32.6]; $P<0.0001$, Mann-Whitney test). Categories showing the most improvements were children's, drinks and fruit yogurts. Fifteen percent of the 2019 products contained less than or equal to $5 \mathrm{~g} / 100 \mathrm{~g}$ sugars, considered a 'low sugar' product, an improvement over the $9 \%$ identified in 2016 . We conclude the sugar content of UK yogurt products has reduced since the sugar reduction program was put into place in 2016. However, a larger reduction in most categories is needed in order to reach the required $20 \%$ reduction by 2020 .
\end{abstract}

\section{Conflict of Interest}

There is no conflict of interest 